\title{
Modelling of T Tauri jets with low mass accretion rate
}

\author{
N. Globus ${ }^{1}$, C. Sauty ${ }^{1}$, V. Cayatte ${ }^{1}$, Z. Meliani ${ }^{1,2}$, J. J. G. Lima ${ }^{3,4}$, \\ K. Tsinganos ${ }^{5}$ and C. Michaut ${ }^{1}$ \\ ${ }^{1}$ LUTh, Observatoire de Paris, F-92190 Meudon, France \\ email: noemie.globus@obspm.fr \\ ${ }^{2}$ Centrum voor Plasma Astrofysica, Leuven, Belgium \\ ${ }^{3}$ Centro de Astrofisica da Universidade do Porto, Portugal \\ ${ }^{4}$ Departamento de Fisica e Astronomia da Faculdade de Ciencias, Porto, Portugal \\ ${ }^{5}$ IASA and Section of Astrophysics, Astronomy \& Mechanics, Department of Physics, \\ University of Athens, Greece
}

\begin{abstract}
We show that low mass accreting $\mathrm{T}$ Tauri stars may have a strong stellar jet component which can effectively brake the star to the observed rotation speed. By means of meridional self similarity, we construct semi analytical solutions describing the complete dynamics and topology of the stellar component of the jet emerging from the corona of the star. We show two typical solutions with the same mass loss rate but different magnetic lever arms and jet radius, corresponding to differente phases of $\mathrm{T}$ Tauri star activity.
\end{abstract}

Keywords. MHD, stars: winds, outflows, stars: mass loss, stars: rotation

\section{Meridionally self-similar model}

The basic equations governing plasma outflows in the framework of ideal MHD for steady, axisymmetric flows are the momentum, mass and magnetic flux conservation, the frozen-in law for infinite conductivity and the first law of thermodynamics. By assuming self-similarity in the meridional $(-\theta)$ direction, we can obtain solutions of the MHD equations. The complete description of the model can be found in Sauty \& Tsinganos (1994). This model presents a double component jet structure for cylindrically collimated solutions. One part of the jet comes from the star itself while the other comes from the inner boundary of the disk which is connected with the stellar magnetosphere.

Disk winds can remove most of the angular momentum of the accreting plasma. However, they cannot describe the inner stellar jet and explain the slowing down of the star itself. Moreover, more evolved T Tauri stars like RY Tau seem to have weaker jets with accretion rates of the order of only a few $10^{-8} M_{\odot} /$ yr. Our aim is to examine the contribution of the stellar component to the overall jet and how efficiently it brakes the star.

\section{Constraining the parameters from the observations}

We have constructed two different solutions with typical values close to the ones observed for RY Tau and other low mass accreting objects for which we have assumed the same initial mass loss rate and stellar parameters. In both cases, the jet radius is defined as the last flux tube connected to the star. The first solution (Solution 1) has a large lever arm, corresponding to our initial guess, and a large opening angle for the jet. This should be close to the observed values for CTTS having a jet, as RY Tau in 2003. This is a solution where the asymptotic structure is collimated only by the magnetic hoop stress. This type of solution gives the largest possible angle for the jet. The star braking 

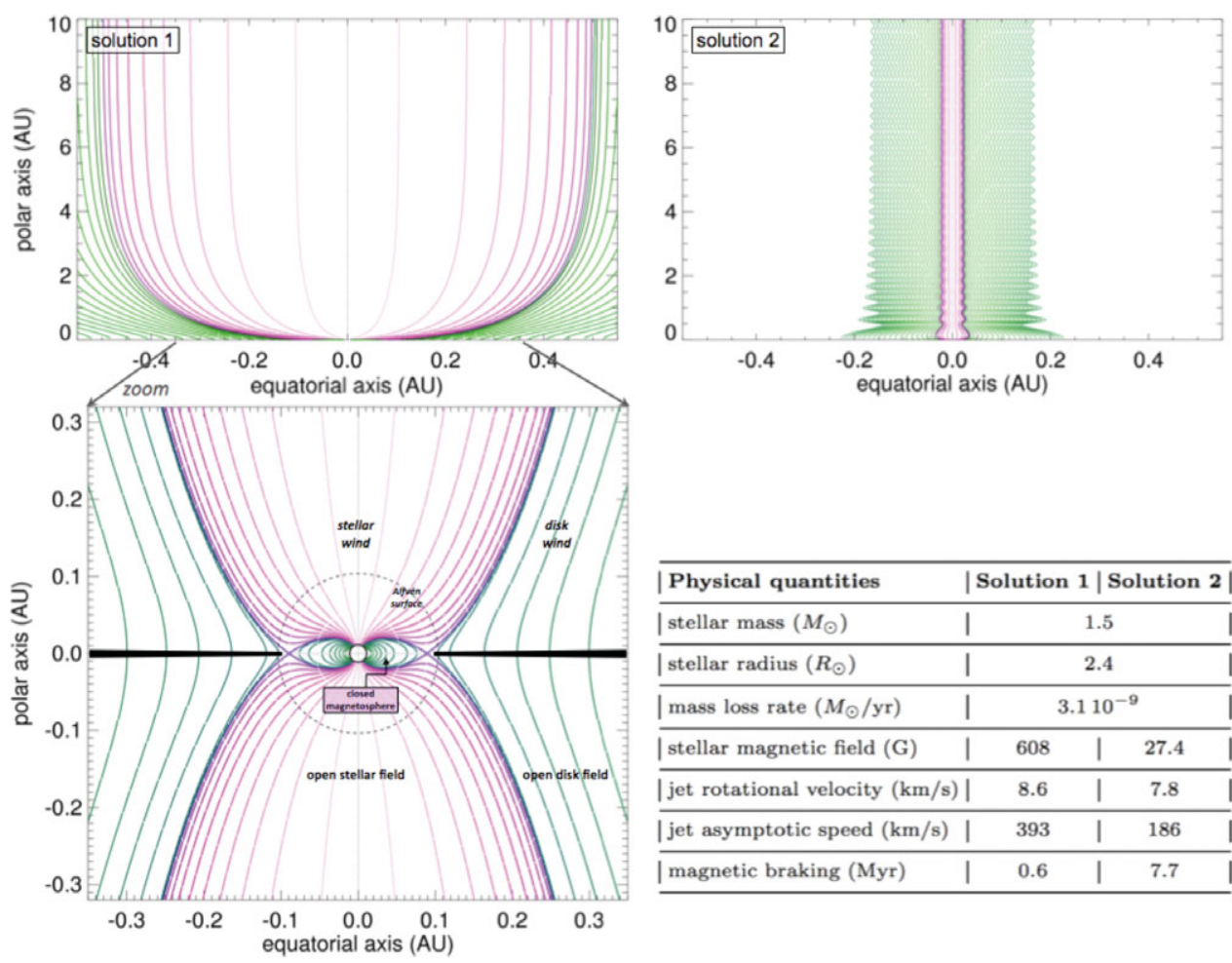

\begin{tabular}{|c|c|c|c|c|c|}
\hline Physical quantities & \multicolumn{5}{|c|}{ | Solution 1 | Solution 2 | } \\
\hline | stellar mass $\left(M_{\odot}\right)$ & I & \multicolumn{4}{|c|}{1.5} \\
\hline | stellar radius $\left(R_{\odot}\right)$ & I & \multicolumn{4}{|c|}{2.4} \\
\hline mass loss rate $\left(M_{\odot} / \mathrm{yr}\right)$ & | & \multicolumn{4}{|c|}{$3.110^{-9}$} \\
\hline stellar magnetic field (G) & I & 608 & I & 27.4 & I \\
\hline jet rotational velocity $(\mathrm{km} / \mathrm{s})$ & & 8.6 & 1 & 7.8 & \\
\hline jet asymptotic speed $(\mathrm{km} / \mathrm{s})$ & 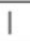 & 393 & $\mathrm{I}$ & 186 & \\
\hline gg (Myr) & 1 & 0.6 & 1 & 7.7 & \\
\hline
\end{tabular}

Figure 1. Topologies of the non-oscillating collimated solution (left panel) and the recollimating solution (right panel). The pink part represents the streamlines connected to the star, i.e. the stellar component of the jet solution. The first solution with a large lever arm and jet radius effectively brakes the star and can be applied to CTTS jets such as the one of RY TAU. The second solution with a smaller lever arm and a very narrow jet may explain why some CTTS do not have visible jets. RY Tau for instance seems to have different phases depending probably on the activity of the star.

time in this case is around $0.610^{6} \mathrm{yr}$, within the typical lifetime for a CTTS $(\approx 1 \mathrm{Myr})$. The initial challenge with modelling RY Tau jet is the presence of a UV shock. We know from previous studies (Pelletier \& Pudritz, 1992) that shocks may occur in our analytical solutions at the recollimating point where the jet starts oscillating. Thus, we have looked for a solution that starts recollimating at the UV shock observed by Gomez \& Verdugo (2001). By slightly changing the parameters we were able to get a completely different solution (Solution 2). The lever arm is reduced as well as the opening angle. The solution exhibits strong recollimation and oscillations. It may either reproduce CTTS such as RY Tau in 2001 when the jet was not visible, but a UV shock was detected close to the star possibly due to the recollimation. In this case the jet is not sufficient to brake the star in the lifetime of a CTTS. It may also correspond to a WTTS (Weak-line T Tauri Star) where jets are not detected. The braking time in this case is around $7.710^{6} \mathrm{yr}$ which is roughly the typical lifetime for a WTTS ( $\approx 10 \mathrm{Myr})$.

\section{References}

Sauty C. \& Tsinganos K. 1994, A\&A, 287, 893

Pelletier G. \& Pudritz R. E. 1992, ApJ, 394, 117

Gomez de Castro A. I. \& Verdugo E. 2001, ApJ, 548, 976 